\title{
Foods and Beverages Associated with Higher Intake of Sugar- Sweetened Beverages
}

\author{
Kevin C. Mathias, MS, Meghan M. Slining, PhD, and Barry M. Popkin, PhD \\ Department of Nutrition, University of North Carolina at Chapel Hill, Chapel Hill, North Carolina
}

\begin{abstract}
Background-Although consumption of sugar-sweetened beverages (SSBs) is associated with higher caloric intakes, the amount SSBs contribute to higher intakes has not been addressed.

Purpose-To estimate the amount SSB contribute to higher caloric intakes and determine how the diets of SSB consumers and nonconsumers differ.

Methods-The WWEI America (What We Eat in America), NHANES 2003-2010 surveys were combined into a sample of 13,421 children; analyses were conducted in December 2012. To determine the contribution of SSB to higher caloric intakes, total non-SSB, food, and non-SSB beverage intakes of SSB consumers and nonconsumers were compared using linear regression models controlling for demographic and socioeconomic factors. Analyses also compared intakes between nonconsumers and SSB consumers with different amounts of SSB consumption.
\end{abstract}

Results-For children aged 2-5 years and 6-11 years, non-SSB intakes did not differ between nonconsumers and SSB consumers at any level of SSB consumption, indicating that SSBs were primarily responsible for the higher caloric intakes among SSB consumers. A similar finding was observed among children aged 12-18 years; however, both food and SSB contribute to higher caloric intakes of adolescents consuming $2500 \mathrm{kcal}$ of SSBs. Among those aged 12-18 years, higher intakes of foods (e.g., pizza, burgers, fried potatoes, and savory snacks) and lower intakes of non-SSB beverages (e.g., fluid milk and fruit juice) were associated with increased SSB intake.

Conclusions-Sugar-sweetened beverages are primarily responsible for the higher caloric intakes of SSB consumers, and SSB consumption is associated with intake of a select number of food and beverage groups, some of which are often unhealthy (e.g., pizza and grain-based desserts).

\section{Introduction}

Increased consumption of sugar-sweetened beverages (SSBs) among children and adolescents is associated with higher caloric intake. ${ }^{1-4}$ Given the increase in SSB consumption over the past 20 years,, 5 the relationship between SSB intake and total energy intake is concerning. To date, the primary focus of SSB-related research has been on SSB intake itself or total energy intake; research is lacking on how overall diet is affected by SSB

(C) 2013 American Journal of Preventive Medicine. Published by Elsevier Inc. All rights reserved.

Address correspondence to: Barry Popkin, PhD, Department of Nutrition, Gillings School of Global Public Health, University of North Carolina at Chapel Hill, University Square, CB\#8120, 123 West Franklin Street, Chapel Hill NC 27516-3997. popkin@unc.edu. No financial disclosures were reported by the authors of this paper.

Publisher's Disclaimer: This is a PDF file of an unedited manuscript that has been accepted for publication. As a service to our customers we are providing this early version of the manuscript. The manuscript will undergo copyediting, typesetting, and review of the resulting proof before it is published in its final citable form. Please note that during the production process errors may be discovered which could affect the content, and all legal disclaimers that apply to the journal pertain. 
intake. This study examines whether increased SSB intake is associated with food ("food" is used to indicate solid food, throughout the paper) and non-SSB beverage intake.

One small study with children aged 6-13 years showed that consumption of any amount of sweetened beverages did not affect food intake, indicating that the sweetened beverages were responsible for increases in total daily energy intake. ${ }^{2}$ Additionally, intake of milk was lower on days when $>12 \mathrm{oz}$. of sweetened drinks were consumed. Consumption of SSBs has also been associated with lower intake of fruit ${ }^{7}$ and with fast-food intake. ${ }^{8}$ Associations between SSB consumption, particular food groups, dietary quality, and micro/ macronutrients have previously been investigated ${ }^{4,7-10}$; however, a comprehensive assessment of foods and beverages associated with SSB consumption has yet to be undertaken. The primary aims of this study were to determine the extent to which SSB contribute to higher caloric intake of SSB consumers and to identify food and beverage groups from the overall diet that are associated with increased SSB consumption.

\section{Methods}

\section{Study Population}

The 2003-2004, 2005-2006, 2007-2008, and 2009-2010 What We Eat in America, National Health and Nutrition Examination Surveys (NHANES) ${ }^{11,12}$ were combined for this analysis; analyses were conducted in December 2012. All surveys were self-weighting, multistage, stratified area probability samples of the non-institutionalized U.S. population. The study population included non-Hispanic whites, non-Hispanic blacks, and Mexican Americans. Non-Mexican-American Hispanics and other races were excluded due to inadequate sample sizes to accurately estimate intake.

The final sample included 10,955 children (aged 2-18 years) with complete data for all covariates included in the models. The sample was stratified into the following age categories: 2-5 years, 6-11 years, and 12-18 years. Dietary intakes for all four surveys were obtained from the Day-1 interviewer-administered 24-hour dietary recall using a multiplepass method. ${ }^{13,14}$

\section{Definition of Sugar-Sweetened Beverages}

Sugar-sweetened beverages were defined as: caloric, sweetened soda; fruit drinks (not including fruit juices); sports drinks; and energy drinks. Milk beverages containing added sugars were not classified as SSBs. Consumers of SSBs were defined as individuals that consumed $>0 \mathrm{kcal}$ of SSBs based on their Day-1 dietary intake assessment.

\section{Definition of Non-Sugar-Sweetened Beverage Calories}

The following formulas were used to determine the contribution of SSBs to the higher caloric intakes of SSB consumers compared to nonconsumers:

- $\quad$ SSB consumer: total caloric intake $=$ food + non-SSB beverages + SSB;

- Nonconsumer: total caloric intake $=$ food + non-SSB beverages;

- Total non-SSB = food + non-SSB beverages.

\section{Food and Beverage Groups}

The food-grouping system used for this paper systematically disaggregated the U.S. Department of Agriculture food groupings based on nutrient composition and critical dietary behaviors. In addition, selected popular foods (e.g., pizza and burgers) that are among the top sources of calories in this study population ${ }^{15}$ were classified as their own group. A total 
of 42 mutually exclusive food groups and eight non-SSB beverage groups were created (Appendix A, available online at www.ajpmonline.org).

\section{Data Analysis}

Analyses were conducted using Stata, version 12.0, and accounted for the complex survey design of the NHANES data set, resulting in estimates representative of the U.S. population. Three linear regression models were used to determine associations between SSB intake and the following dietary outcome variables: total intake (kcal); non-SSBs (kcal); non-SSB beverages ( $\mathrm{kcal})$; food ( $\mathrm{kcal})$; and food energy density (kcal/gram). In the first model, SSB consumption was coded as a dichotomous variable to test differences between SSB consumers and nonconsumers. The second model included only SSB consumers and examined the linear relationship between increases in SSB intake and dietary intake variables.

A nonlinear relationship between non-SSB intake and SSB consumption (including nonconsumers) was observed; therefore, SSB consumers were divided into three categories (low, medium, and high), and intakes for these categories were compared to the nonconsumers in the third model. The three categories were chosen by visually examining the relationship of non-SSB intakes versus SSB consumption in increments of $100 \mathrm{kcal}$ of SSB. Cut-points for the categories were chosen to best depict the shape of the relationship between non-SSB and SSB intake. Because the relationship between non-SSB intake and SSB intake was different for each of the three age groups, different cut-points were chosen for the SSB consumer categories.

All models were adjusted for age; gender; weight $(\mathrm{kg})$; race/ethnicity (non-Hispanic white, non-Hispanic black, Mexican-American); household income ( $₫ 85 \%, 186 \%-300 \%$, and $>300 \%$ of poverty level); and weekend (dietary assessment taken from a weekend or weekday). Interactions between race/ethnicity and SSB intake were tested for each age group in all three regression models.

Having a large number of individuals who do not consume a particular food/beverage group violates normality assumptions for estimating per capita intake using OLS models; therefore, two-stage marginal effects models were conducted. The first stage uses a probit model to determine the probability that an individual in a given SSB consumption group consumed a particular food/beverage group. The second stage uses a linear regression model to determine the intake of a particular food/beverage group only among consumers of that food/beverage group. The marginal effects model takes into account the results from both models to compute differences in intake between the SSB consumption categories and the referent nonconsumer group. The criterion used for all tests of significance was $p<0.05$.

\section{Results}

The socioeconomic, demographic, and per capita intakes (kcal) of food, SSBs, and non-SSB beverages for the three age categories are presented in Table 1. For the three age groups, SSB consumers had higher $(p<0.0001)$ total caloric intakes than nonconsumers; $+166 \pm 32$, $+240 \pm 34$, and $+359 \pm 47 \mathrm{kcal}$ for children aged $2-5$ years, $6-11$ years, and $12-18$ years, respectively (data not shown). In order to determine the contribution of SSB to higher caloric intake, non-SSB intakes were examined.

\section{Children Aged 2-5 Years}

Intake of non-SSB calories did not differ $(+21 \pm 31 \mathrm{kcal})$ between SSB consumers and nonconsumers (data not shown). Categorizing SSB consumer status as simply consumer or nonconsumer may not fully capture the heterogeneity among SSB consumers; therefore, a 
secondary approach was taken to determine the relationship between intake of calories from non-SSB sources and amount of SSB consumption using only SSB consumers. Because changes in food and non-SSB beverage consumption affect non-SSB intake, both sources of calories were examined. Higher SSB consumption was associated with increased food intake, but with lower non-SSB beverage intake, resulting in no association between SSB intake and non-SSB calories (Table 2).

An interaction was observed between race/ethnicity and SSB consumption with respect to non-SSB beverage intake. Both non-Hispanic blacks and Mexican Americans significantly decreased their intake of non-SSB beverages by $23 \pm 6$ and $49 \pm 10$ per 100-kcal increase in SSB, whereas, non-Hispanic whites did not significantly decrease their intakes (Appendix B, available online at www.ajpmonline.org). Intake of non-SSB calories did not differ across the levels of SSB consumption (Table 3). These findings indicate that for children aged 2-5 years, SSB consumption at any level is the main reason for higher caloric intake compared to that of nonconsumers.

\section{Children Aged 6-11 Years}

Intake of non-SSB calories did not differ $(17 \pm 39 \mathrm{kcal})$ between SSB consumers and nonconsumers (data not shown). Among SSB consumers, intake of non-SSB beverage calories was not associated with increased SSB intake; however, food intake increased by 36 \pm 14 kcal per 100-kcal SSB increase (Table 2). An interaction was observed between race/ ethnicity and SSB consumption with respect to food intake. Intake of food (kcal) among non-Hispanic whites was not associated with SSB consumption. By contrast, both nonHispanic blacks and Mexican Americans had increased intake of food (kcal) with increased SSB intake: $52 \pm 21$ and $94 \pm 13 \mathrm{kcal}$ of food per 100-kcal increase of SSBs, respectively (Appendix B, available online at www.ajpmonline.org).

Among SSB consumers, only those with the highest levels of consumption ( $\geq 200 \mathrm{kcal}$ ) had higher food intake; all SSB consumer groups had lower non-SSB beverage intakes than nonconsumers (Table 3). Intake of non-SSB calories did not differ across levels of SSB consumption. This result indicates that for children aged 6-11 years, any amount of SSB consumption is the primary reason for the higher caloric intakes of SSB consumers as compared to nonconsumers.

\section{Children Aged 12-18 Years}

An interaction was observed between race/ethnicity and differences between SSB consumers and nonconsumers with respect to non-SSB intake; $-25 \pm 66,83 \pm 68$, and $177 \pm$ $61 \mathrm{kcal}$ for non-Hispanic whites, non-Hispanic blacks, and Mexican Americans, respectively (data not shown). Among SSB consumers aged 12-18 years, intake of food increased by 86 $\pm 10 \mathrm{kcal}$ for every 100-kcal increase in SSB intake, and non-SSB beverage intakes decreased by $9 \pm 2$ for every 100-kcal increase in SSB intake (Table 2). As a result, when only SSB consumers were analyzed in the model, only those aged 12-18 years showed a positive association of intake of non-SSB calories with SSB intake.

In the categoric analysis, an interaction was observed between race/ethnicity and differences between nonconsumers and each category of SSB consumption for the following outcome variables: total intake, non-SSB intake, non-SSB beverage and food intake (Appendix C, available online at www.ajpmonline.org). The main factor driving this interaction was intake of food (kcal) among nonconsumers, where non-Hispanic blacks and Mexican Americans consumed $1600 \pm 62$ and $1469 \pm 66 \mathrm{kcal}$, respectively, and non-Hispanic whites consumed $1751 \pm 47 \mathrm{kcal}$. Across all races/ethnicities, intake of non-SSB beverages was lower and 
food energy density (kcal/gram) was higher for medium (100 2SSB kcal >500) and high ( $2500 \mathrm{kcal}$ ) SSB consumers compared to nonconsumers.

For all races/ethnicities, high SSB consumers ( $2500 \mathrm{kcal} ; 13 \%$ of children aged $12-18$ years) consumed more non-SSB calories than nonconsumers, indicating that both food and SSBs contributed to higher caloric intakes among individuals consuming $2500 \mathrm{kcal}$ of SSBs (Appendix C, available online at www.ajpmonline.org). Among non-Hispanic blacks and Mexican Americans, both food and SSBs contributed to the higher caloric intakes of medium SSB consumers (100-499 kcal SSB) as well. Fifty-six percent of non-Hispanic blacks and 58\% of Mexican Americans in this age group consumed 100-499 kcal of SSBs.

\section{Food and Beverage Groups Associated with Intake of Sugar-Sweetened Beverages}

Across all age groups, the energy density (kcal/gram) of food consumed increased with higher SSB consumption (Tables 2 and 3); however, of the 50 food/beverage groups tested in the current analysis, only a few significant differences of $>10 \mathrm{kcal}$ were observed between nonconsumers and low, medium and high SSB consumers (Appendixes D-F, available online at www.ajpmonline.org). Appendixes D-F report only the foods and beverages for which a significant difference of $>10 \mathrm{kcal}$ was observed between nonconsumers (reference group) and one of the SSB consumption categories.

The results below report the largest differences in food and beverage intakes between nonconsumers and SSB consumers. Medium SSB consumers consumed more pizza (kcal) than nonconsumers among children aged $12-18$ years $(53 \pm 21 \mathrm{kcal})$. For this age group, the highest SSB consumers ( $2500 \mathrm{kcal}$ ) consumed $118 \pm 36 \mathrm{kcal}$ more pizza, $104 \pm 22 \mathrm{kcal}$ more burgers, $60 \pm 12 \mathrm{kcal}$ more fried potatoes, and $43 \pm 19 \mathrm{kcal}$ more savory snacks than nonconsumers.

Across all age groups, decreases in fruit intake (kcal) were observed among medium and high SSB consumers. Across all age groups, decreases in fruit juice intake (kcal) were observed for all three SSB consumption groups. Only among children aged 2-5 years was there was a decrease in fluid milk consumption across all levels of SSB consumption; among those aged 6-11 years, only the highest SSB consumers ( $\geq 200 \mathrm{kcal}$ ) consumed less fluid milk than non-consumers, and among those aged 12-18 years, only SSB consumers $(100+\mathrm{kcal})$ did so.

Grain-based dessert (e.g., cakes, cookies and pies) intake was $44 \pm 14$ and $36 \pm 17 \mathrm{kcal}$ higher among the highest ( $\geq 200 \mathrm{kcal}$ ) SSB consumers for children aged 2-5 years and 6-11 years, respectively. Only among those aged $2-5$ years were calories of sweet snacks (e.g., candy) associated with SSB intake. In this age group, intake was higher for all SSB consumption groups compared to nonconsumers.

\section{Discussion}

Higher consumption of SSBs was associated with higher food intake among SSB consumers, offset partially by reductions in non-SSB beverage intake. Among children aged 2-5 years and 6-11 years, intake of non-SSB calories did not differ across levels of SSB consumption, indicating that SSB intake was primarily responsible for the higher caloric intakes of SSB consumers as compared to nonconsumers. Of particular interest are those aged 12-18 years who consume 500 or more kcal from SSBs, and non-Hispanic blacks and Mexican Americans in the same age group who consume 100-499 kcal SSBs. For these groups, both food and SSBs contributed to higher caloric intake. 
Among all age groups analyzed, the energy density of food consumed increased as SSB intake increased. Energy-density shifts of the magnitude found here have been linked to higher energy intake among adults. ${ }^{16-19}$ Given these findings, future research on the effects of dietary energy density and total caloric intake should account for these associations between total caloric intake, SSB intake, and food energy density. Examinations of overall diets showed that only a small number of foods and beverages were associated with SSB intake. The largest associations were decreases in fluid milk among those aged 2-5 years and increases in intake of pizza, burgers, and fried potatos among the highest ( $2500 \mathrm{kcal}$ ) SSB consumers aged $12-18$ years.

Few studies have taken this unique approach of examining non-SSB intake to determine the extent to which SSBs contribute to higher caloric intake. One study with 30 children aged 613 years showed that total calories increased with higher consumption of sweetened beverages, but intake (kcal) of food was similar across all levels of sweetened beverage consumption. ${ }^{2}$ Mrdjenovic et al. concluded that because food intake remained constant when sweetened beverage intake was increased, higher energy intakes were entirely the result of higher sweetened beverage consumption.

The current study results led to a similar conclusion, but showed that across all age groups, intake of food (kcal) was higher among the highest SSB consumers,' and intake of non-SSB beverage calories was lower, compared to nonconsumers. However, only among those aged 12-18 years did the highest consumers of SSB consume significantly more non-SSB calories than nonconsumers. As a result, the higher caloric intake of this group can be attributed to both SSB and higher food intake. To the author's knowledge, this is the first study to show the extent to which SSB, food, and non-SSB beverage intakes contribute to higher caloric intakes of SSB consumers as compared to nonconsumers.

Although previous work in adults has examined associations between food and beverage patterns, ${ }^{20}$ the major focus in children with respect to associations with SSB intake has been on nutrient intakes and overall dietary quality. ${ }^{4,7-10}$ To date, associations between SSB intake and consumption of food groups from an overall dietary perspective have not been examined. The results from the current analysis showed that the energy density of food was positively associated with SSB intake; however, only a few significant and meaningful ( $\geq 10$ $\mathrm{kcal}$ ) differences in intake of food/beverage groups were observed between nonconsumers and low, medium and high SSB consumers.

A concerning aspect of these findings is that many of the foods (e.g., pizza, grain-based desserts, fried potatoes, and sweets) that are associated with higher SSB consumption are listed by the 2010 Dietary Guidelines as foods that Americans should limit in their diets. ${ }^{21}$ These foods are some of the top sources of solid fats and added sugars among children and adolescents. ${ }^{22}$ Reducing their intake has been recommended as a means to increase consumption of foods high in micronutrients while limiting excessive caloric intake. ${ }^{21}$

\section{Limitations}

There are important limitations of this study due to the nature of the design. An inability among SSB consumers to compensate for higher beverage intakes with lower food intakes cannot be established with cross-sectional data. In addition, the SSB consumers identified in this study were only consumers for that particular day; therefore, the results should not be generalized to behaviors of all SSB consumers, but rather to consumers of SSB on a given day. Future research is needed to better understand if SSB consumers are inherently different than nonconsumers, or if certain foods and/or circumstances that affect dietary behaviors (e.g., working late, birthday parties, fast-food beverage sizes) are paired with SSB consumption. 


\section{Conclusion}

These results show that consumption of SSBs is primarily responsible for the higher caloric intakes of SSB consumers as compared to individuals that did not consume SSBs. This is the first study to report the extent to which SSB, food, and non-SSB beverage intakes contribute to higher caloric intakes of SSB consumers as compared to individuals that did not consume SSBs. Of particular interest are adolescents who consumed 500 or more kcal of SSBs (13\% of adolescents) and adolescent non-Hispanic blacks and Mexican Americans who consumed $100-499 \mathrm{kcal}$ SSB (55\% of the individuals in these race/ethnicity groups). These newly identified subpopulations represent large groups in which calories from both foods and SSBs contributed to' higher caloric intakes of SSB consumers.

After analyzing the overall dietary patterns of SSB consumers and nonconsumers, only a few food and beverage groups emerged where differences were observed between levels of SSB intake. The key associations were observed among high SSB consumers with lower consumption of fluid milk, fruit juice and fruit; and higher consumption of foods that are among the top sources of solid fats and added sugars. The implications of these findings are that SSBs are primarily responsible for the higher caloric intakes of SSB consumers, and that SSB consumption is associated with the intake of a select number of food and beverage groups, some of which are often unhealthy.

\section{Supplementary Material}

Refer to Web version on PubMed Central for supplementary material.

\section{Acknowledgments}

The authors thank the Robert Wood Johnson Foundation (Grant 67506) and the NIH (R01 HL104580, 1T32HD057824-02 and CPC 5 R24 HD050924) for financial support. The authors also thank Dr. Phil Bardsley for exceptional assistance with the data management and programming, Dr. Shu Wen Ng for programming and modeling assistance, Ms. Frances L. Dancy for administrative assistance, and Tom Swasey for graphical support.

\section{References}

1. Tordoff MG, Alleva AM. Effect of drinking soda sweetened with aspartame or high-fructose corn syrup on food intake and body weight. Am J Clin Nutr. 1990; 51(6):963-9. [PubMed: 2349932]

2. Mrdjenovic G, Levitsky DA. Nutritional and energetic consequences of sweetened drink consumption in 6- to 13-year-old children. J Pediatr. 2003; 142(6):604-10. [PubMed: 12838186]

3. O'Connor TM, Yang SJ, Nicklas TA. Beverage intake among preschool children and its effect on weight status. Pediatrics. 2006; 118(4):e1010-8. [PubMed: 17015497]

4. Harnack L, Stang J, Story M. Soft drink consumption among U.S. children and adolescents: nutritional consequences. J Am Diet Assoc. 1999; 99(4):436-41. [PubMed: 10207395]

5. Lasater G, Piernas C, Popkin BM. Beverage patterns and trends among school-aged children in the U.S 1989-2008. Nutr J. 2011; 10:103. [PubMed: 21962086]

6. Popkin BM. Patterns of beverage use across the lifecycle. Physiol Behav. 2010; 100(1):4-9. [PubMed: 20045423]

7. Frary CD, Johnson RK, Wang MQ. Children and adolescents' choices of foods and beverages high in added sugars are associated with intakes of key nutrients and food groups. J Adolesc Health. 2004; 34(1):56-63. [PubMed: 14706406]

8. Bowman SA, Gortmaker SL, Ebbeling CB, Pereira MA, Ludwig DS. Effects of fast-food consumption on energy intake and diet quality among children in a national household survey. Pediatrics. 2004; 113(1):112-8. [PubMed: 14702458]

9. Marshall TA, Eichenberger, Gilmore JM, Broffitt B, Stumbo PJ, Levy SM. Diet quality in young children is influenced by beverage consumption. J Am Coll Nutr. 2005; 24(1):65-75. [PubMed: 15670987] 
10. Ballew C, Kuester S, Gillespie C. Beverage choices affect adequacy of children's nutrient intakes. Arch Pediatr Adolesc Med. 2000; 154(11):1148-52. [PubMed: 11074858]

11. NHANES Analytic and Reporting Guidelines. CDC; 2005. www.cdc.gov/nchs/data/nhanes/ nhanes_03_04/nhanes_analytic_guidelines_dec_2005.pdf

12. NHANES Dietary Web Tutorial. NHANES Dietary Data Collection. CDC; Dietary Data Overview. www.cdc.gov/nchs/tutorials/Dietary/SurveyOrientation/DietaryDataOverview/ Info2.htm

13. Moshfegh AJ, Rhodes DG, Baer DJ, et al. The U.S Department of Agriculture Automated Multiple-Pass Method reduces bias in the collection of energy intakes. Am J Clin Nutr. 2008; 88(2):324-32. [PubMed: 18689367]

14. Blanton CA, Moshfegh AJ, Baer DJ, Kretsch MJ. The USDA Automated Multiple-Pass Method accurately estimates group total energy and nutrient intake. J Nutr. 2006; 136(10):2594-9. [PubMed: 16988132]

15. Applied Research Program. National Cancer Institute; 2010. Sources of Energy among the U.S. Population, 2005-06. Risk Factor Monitoring and Methods Branch Web site. riskfactor.cancer.gov/diet/foodsources/energy/table1a.html

16. Ledikwe JH, Rolls BJ, Smiciklas-Wright H, et al. Reductions in dietary energy density are associated with weight loss in overweight and obese participants in the PREMIER trial. Am J Clin Nutr. 2007; 85(5):1212-21. [PubMed: 17490955]

17. Bell EA, Castellanos VH, Pelkman CL, Thorwart ML, Rolls BJ. Energy density of foods affects energy intake in normal-weight women. Am J Clin Nutr. 1998; 67(3):412-20. [PubMed: 9497184]

18. Ledikwe JH, Blanck HM, Kettel Khan L, et al. Dietary energy density is associated with energy intake and weight status in U.S. adults. Am J Clin Nutr. 2006; 83(6):1362-8. [PubMed: 16762948]

19. Rolls BJ. Plenary Lecture 1: Dietary strategies for the prevention and treatment of obesity. Proc Nutr Soc. 2010; 69(1):70-9. [PubMed: 19954563]

20. Duffey KJ, Popkin BM. Adults with healthier dietary patterns have healthier beverage patterns. J Nutr. 2006; 136(11):2901-7. [PubMed: 17056820]

21. U.S. Department of Agriculture and DHHS. Dietary Guidelines for Americans, 2010. 7. U.S. Government Printing Office; Washington, DC: Dec. 2010

22. Reedy J, Krebs-Smith SM. Dietary sources of energy, solid fats, and added sugars among children and adolescents in the U.S. J Am Diet Assoc. 2010; 110(10):1477-84. [PubMed: 20869486] 


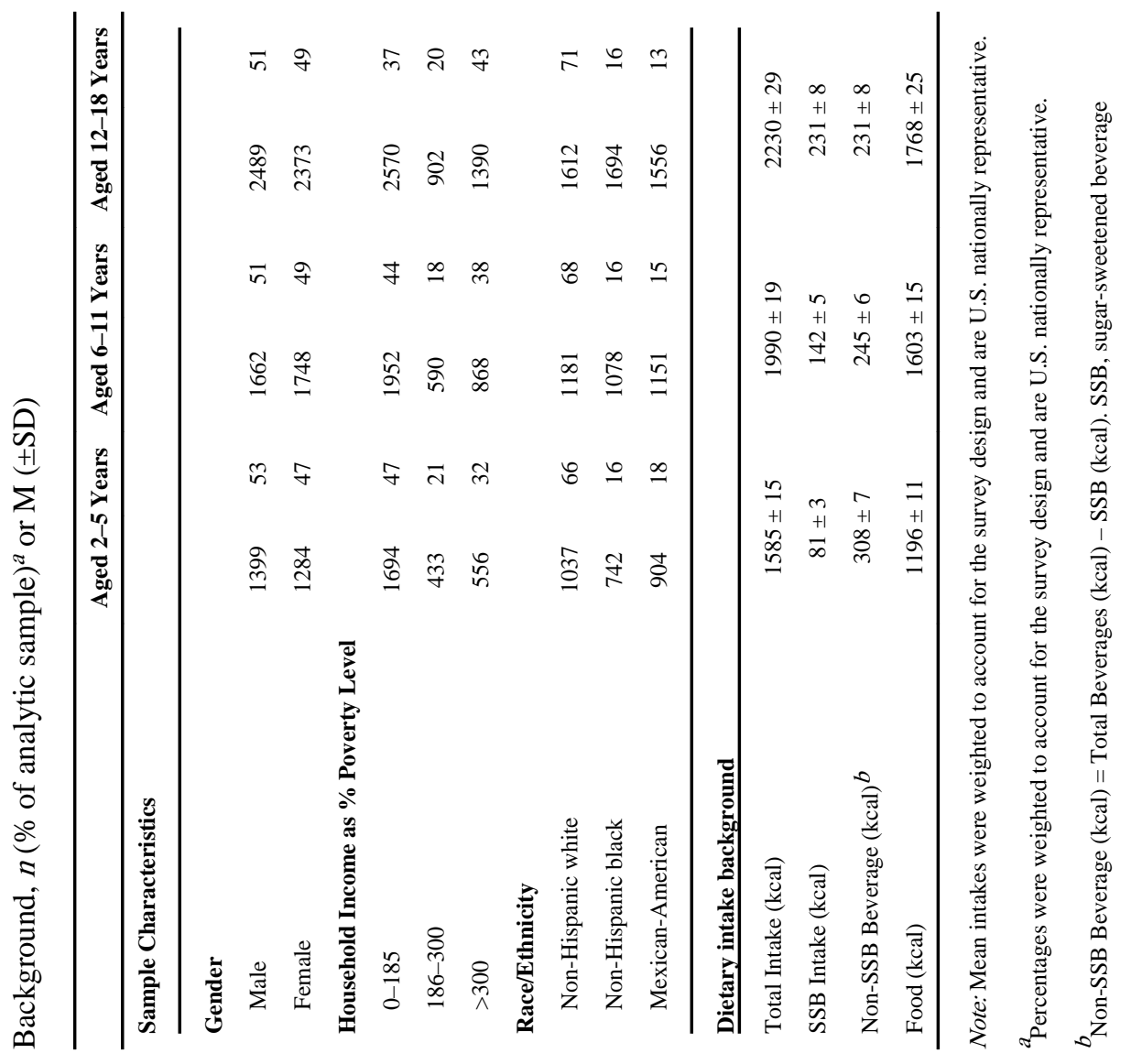


Table 2

Associations between sugar-sweetened beverage (SSB) consumption and caloric intake among SSB consumers

\begin{tabular}{|c|c|c|c|}
\hline Energy, Nutrients & Aged 2-5 Years & Aged 6-11Years & Aged 12-18 Years \\
\hline & \multicolumn{3}{|c|}{ Change per 100-kcal increase in SSB consumption } \\
\hline Total Intake (kcal) & $+143 \pm 23 *$ & $+127 \pm 15^{*}$ & $+177 \pm 11^{*}$ \\
\hline Total Non-SSB (kcal) ${ }^{a}$ & $+43 \pm 23$ & $+27 \pm 15$ & $+77 \pm 11^{*}$ \\
\hline Non-SSB Beverage $(\mathrm{kcal})^{b}$ & $-20 \pm 7^{*}$ & $-9 \pm 6$ & $-9 \pm 2^{*}$ \\
\hline Food (kcal) & $+63 \pm 19^{*}$ & $+36 \pm 14^{*}$ & $+86 \pm 10^{*}$ \\
\hline Food Energy Density $(\mathrm{kcal} / \mathrm{gram})^{c}$ & $0.06 \pm 0.02 *$ & $0.04 \pm 0.01 *$ & $0.02 \pm 0.007 *$ \\
\hline
\end{tabular}

Note: Boldface indicates significance. Estimates were obtained from linear regression models for each age group separately where the estimated coefficients represent the change in the outcome (e.g., total intake) per 100-kcal increase in SSB consumption. All models adjusted for age, gender, race/ethnicity, weight $(\mathrm{kg})$, household income, and weekend (whether the dietary assessment was from a weekday or weekend)

${ }^{a}$ Total Non-SSB $(\mathrm{kcal})=$ Total Energy $(\mathrm{kcal})-$ SSB $(\mathrm{kcal})$

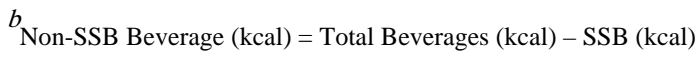

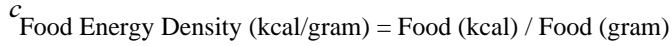

* Coefficients for estimates of slope are significantly different from zero $(p<0.05)$. 
Table 3

Energy intakes by category of sugar-sweetened beverage (SSB) consumption

\begin{tabular}{|c|c|c|c|c|}
\hline Aged 2-5 years & 0 SSB kcal & $0>$ SSB kcal $>100$ & $100 \geq$ SSB kcal $>200$ & $\geq 200 \mathrm{kcal}$ \\
\hline$\%$ of Age Group & 46 & 24 & 18 & 12 \\
\hline Total Intake (kcal) & $1504 \pm 23$ & $1552 \pm 21$ & $1661 \pm 43^{*}$ & $1854 \pm 52^{*}$ \\
\hline Non-SSB $(\mathrm{kcal})^{a}$ & $1504 \pm 23$ & $1488 \pm 21$ & $1516 \pm 43$ & $1519 \pm 46$ \\
\hline Non-SSB Beverage $(\mathrm{kcal})^{b}$ & $355 \pm 12$ & $297 \pm 11^{*}$ & $269 \pm 14^{*}$ & $206 \pm 15^{*}$ \\
\hline Food (kcal) & $1149 \pm 18$ & $1191 \pm 22$ & $1248 \pm 35^{*}$ & $1314 \pm 40^{*}$ \\
\hline Food Energy Density $(\mathrm{kcal} / \text { gram })^{c}$ & $1.94 \pm 0.03$ & $2.10 \pm 0.04^{*}$ & $2.16 \pm 0.05^{*}$ & $2.29 \pm 0.05^{*}$ \\
\hline \multicolumn{5}{|l|}{ Aged 6-11 years } \\
\hline$\%$ of Age Group & 32 & 18 & 23 & 27 \\
\hline Total Intake (kcal) & $1837 \pm 32$ & $1874 \pm 30$ & $1963 \pm 30^{*}$ & $2269 \pm 43^{*}$ \\
\hline Non-SSB $(\mathrm{kcal})^{a}$ & $1836 \pm 32$ & $1802 \pm 30$ & $1818 \pm 30$ & $1918 \pm 42$ \\
\hline Non-SSB Beverage (kcal) $b$ & $283 \pm 11$ & $253 \pm 10^{*}$ & $230 \pm 12 *$ & $208 \pm 10^{*}$ \\
\hline Food (kcal) & $1553 \pm 30$ & $1549 \pm 29$ & $1587 \pm 27$ & $1710 \pm 39^{*}$ \\
\hline Food Energy Density (kcal/gram) ${ }^{\mathcal{C}}$ & $2.08 \pm 0.03$ & $2.16 \pm 0.04$ & $2.30 \pm 0.03 *$ & $2.35 \pm 0.03 *$ \\
\hline Aged 12-18 years & 0 SSB kcal & 0 > SSB kcal > 100 & $100 \leq \mathrm{SSB}$ kcal $>500$ & $\succeq 00 \mathrm{kcal}$ \\
\hline$\%$ of Age Group & 30 & 7 & 50 & 13 \\
\hline Total Intake (kcal) & $1982 \pm 41$ & $1850 \pm 73$ & $2231 \pm 36^{*}$ & $3035 \pm 89^{*}$ \\
\hline Non-SSB $(k c a l)^{\mathrm{s}}$ & $1979 \pm 41$ & $1779 \pm 72 *$ & $1967 \pm 36$ & $2301 \pm 81^{*}$ \\
\hline Non-SSB Beverage $(\mathrm{kcal})^{b}$ & $287 \pm 13$ & $232 \pm 17^{*}$ & $211 \pm 10^{*}$ & $175 \pm 19^{*}$ \\
\hline Food (kcal) & $1692 \pm 37$ & $1546 \pm 67^{*}$ & $1756 \pm 32$ & $2125 \pm 71^{*}$ \\
\hline Food Energy Density $(\mathrm{kcal} / \mathrm{gram})^{c}$ & $2.13 \pm 0.03$ & $2.24 \pm 0.08$ & $2.35 \pm 0.02^{*}$ & $2.45 \pm 0.04 *$ \\
\hline
\end{tabular}

Note: Boldface indicates significance. Estimates were generated from linear regression models for each age category separately using SSB consumption as an indicator variable. Comparisons were made between each level of SSB consumption and the referent nonconsumer group at a significance level of $p<0.05$. All models were adjusted for age, gender, race/ethnicity, weight (kg), household income, and weekend (whether the dietary assessment was from a weekday or weekend).

${ }^{a}$ Non-SSB $(\mathrm{kcal})=$ Total Energy $(\mathrm{kcal})-\mathrm{SSB}(\mathrm{kcal})$

${ }^{b}$ Non-SSB Beverage $(\mathrm{kcal})=$ Total Beverage $(\mathrm{kcal})-\mathrm{SSB}(\mathrm{kcal})$

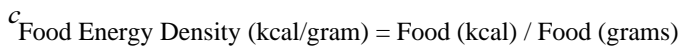

Indicates a significant difference $(p<0.05)$ between an SSB consumption group and the reference nonconsumer group

SSB, sugar-sweetened beverage 